\title{
Individual-, maternal- and household-level factors associated with stunting among children aged 0-23 months in Bangladesh
}

\author{
Sabui Kanti Mistry ${ }^{1, *}$, Md. Belal Hossain ${ }^{1}$, Fouzia Khanam ${ }^{1}$, Fahmida Akter ${ }^{1}$, \\ Mahmood Parvez ${ }^{1}$, Fakir Md Yunus ${ }^{2}$, Kaosar Afsana ${ }^{3}$ and Mahfuzar Rahman ${ }^{1}$ \\ ${ }^{1}$ Research and Evaluation Division, BRAC, BRAC Centre, 75 Mohakhali, Dhaka 1212, Bangladesh: ${ }^{2}$ College of \\ Pharmacy and Nutrition, The University of Saskatchewan, Saskatoon, SK, Canada: ${ }^{3}$ Health Nutrition and Population \\ Programme, BRAC, Dhaka, Bangladesh
}

Submitted 28 March 2018: Final revision received 21 September 2018: Accepted 25 September 2018: First published online 8 November 2018

\begin{abstract}
Objective: Childhood stunting remains a major public health concern in Bangladesh. To accelerate the reduction rate of stunting, special focus is required during the first 23 months of a child's life when the bulk of growth takes place. Therefore the present study explored individual-, maternal- and household-level factors associated with stunting among children under 2 years of age in Bangladesh.

Design: Data were collected through a nationwide cross-sectional survey conducted between October 2015 and January 2016. A two-stage cluster random sampling procedure was applied to select 11428 households. In the first stage, 210 enumerations areas (EA) were selected with probability proportional to EA size (180 EA from rural areas, thirty EA from urban slums). In the second stage, an average of fifty-four households were selected from each EA through systematic random sampling.

Setting: Rural areas and urban slums of Bangladesh.

Participants: A total of 6539 children aged 0-23 months.

Results: Overall, $29.9 \%$ of the children were stunted. After adjusting for all potential confounders in the modified Poisson regression model, child's gender, birth weight (individual level), maternal education, age at first pregnancy, nutrition (maternal level), administrative division, place of residence, socio-economic status, food security status, access to sanitary latrine and toilet hygiene condition (household level) were significantly associated with stunting.

Conclusions: The study identified a number of potentially addressable multilevel risk factors for stunting among young children in Bangladesh that should be addressed through comprehensive multicomponent interventions.
\end{abstract}

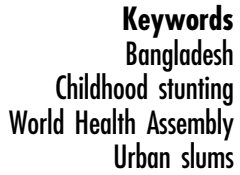

Keywords

Childhood stunting

Urban slums
Linear growth faltering in childhood, also familiarly known as stunting, is a major global health concern ${ }^{(1)}$. Stunting has both short-term and long-term consequences on health and development throughout the life cycle ${ }^{(2-4)}$ as well as across generations ${ }^{(5)}$. Nearly half of all deaths among children under 5 years of age (under- $5 \mathrm{~s}$ ) are attributable to undernutrition, which has both direct and indirect impacts on economic productivity and growth ${ }^{(6)}$. It has been estimated that stunting can reduce a country's gross domestic product by up to $3 \%^{(7)}$ and that as adults, stunted children earn $20 \%$ less than the non-stunted ${ }^{(8)}$.

The causes of stunting are multisectoral and multifactorial. The WHO conceptual framework on childhood stunting describes the complex interaction of household characteristics; water, sanitation and hygiene; environmental issues; socio-economic status; and cultural influences with childhood stunting ${ }^{(4)}$. Causes of malnutrition in early childhood have also been extensively analysed by both Fenske et al. ${ }^{(9)}$ and Goudet et al. ${ }^{(10)}$. These authors have classified the determinants of childhood stunting into immediate (individual level), intermediate (individual/household level) and underlying (maternal, household and regional level) factors.

Globally, although the prevalence of stunting is dropping, there are still around 156 million under-5s who are stunted $^{(11)}$. In 2015 , Asia was the home to $56 \%$ of all stunted under -5 children, while Africa's share was $37 \%{ }^{(11)}$. The prevalence of stunting among under- $5 \mathrm{~s}$ has decreased by an average of $2.7 \%$ per year (from 51 to $36 \%$ ) between 2004 and 2014 in Bangladesh ${ }^{(12)}$. This is far below the 
targeted annual reduction of $3 \cdot 3 \%$, necessary to meet the World Health Assembly target of $40 \%$ reduction by 2025 . Therefore, Bangladesh is less likely to achieve the World Health Assembly target for reducing childhood stunting with the current pace of reduction ${ }^{(6)}$.

Bangladesh has experienced rapid urbanization in recent years and is expected to have more than $50 \%$ urban population by $2050^{(13)}$. As a result, more people are residing in urban slum settlements - a number currently estimated to be $2 \cdot 2$ million $^{(14,15)}$. Congested living conditions, poverty, lack of knowledge on nutrition, and improper hygiene and sanitation facilities all make slum dwellers more vulnerable to recurrent infections which result in health and nutritional deprivation ${ }^{(16,17)}$. Evidence also suggests that the prevalence of stunting among under$5 \mathrm{~s}$ is higher among children living in these slums compared with the overall urban population of Bangladesh $(40 \text { v. } 26 \%)^{(18)}$.

Given the situation, careful exploration of the most important determinants of stunting is critical for governments and development partners. This would enable them to make strategic decisions to reduce the stunting prevalence as well as to plan, design and monitor effective interventions $^{(12,19)}$. However, only a few studies have been conducted to date focusing on comprehensively explaining the myriad factors associated with childhood stunting ${ }^{(19-22)}$. Furthermore, it is broadly accepted that the first $1000 \mathrm{~d}$ from conception through the first 2 years of life is the most critical period for stunting ${ }^{(3,23)}$. Therefore, the present study was carried out to identify the risk factors of stunting at different levels (i.e. individual, maternal and household) among Bangladeshi children aged 0-23 months.

\section{Methods}

\section{Study design and participants}

The data used for the present study were part of a nationwide cross-sectional health-care survey carried out between October 2015 and January 2016. The base survey was conducted by Building Resources Across Communities (BRAC) to explore the overall health status of children, mothers and senior citizens of rural areas and urban slums in Bangladesh. The survey excludes urban non-slum areas as BRAC operates its health interventions in rural areas and urban slums only. A slum is defined as a cluster of compact settlements of five or more households grown unsystematically and haphazardly on government and/or private vacant land ${ }^{(14)}$. A two-stage cluster random sampling procedure was applied and a total of 11428 households (response rate 94.9\%) having at least one under- 5 child was selected in the base survey. In the first stage, 210 enumeration areas (EA) were selected randomly with probability proportional to EA size, with 180 EA from rural areas and thirty EA from urban slums. An EA is a union (rural areas) or ward (urban slums), which is the lowest administrative unit in Bangladesh. A complete list of unions and wards, collected from the Bangladesh Bureau of Statistics, was used as the sampling frame for the first stage of sampling. In the second stage, starting from the north-west corner of an EA with systematic random sample of five households, on average, fifty-four households were selected per EA to provide statistically reliable estimates for rural areas and urban slums separately. Finally, the random selection covered all administrative divisions and fifty-eight out of sixty-four districts of Bangladesh. If a household contained more than one under-5 child, the smallest child was included in the survey. The present analysis was performed among all the children ( $n$ 6539) aged 0-23 months of the selected households (Fig. 1).

\section{Data collection tools and techniques}

A pre-tested structured questionnaire was administered to gather information pertaining to the child, mother and household through face-to-face interview with the child's mother. Anthropometric measurements were taken using standardized procedures. Data collection was carried out electronically in ODK (Open Data Kit), an Android-based open-source mobile platform software ${ }^{(24)}$.

Skilled female interviewers (with health survey experience) were recruited to minimize reporting bias as the respondents were women ${ }^{(25)}$. Fifteen days of intensive training was provided to the interviewers which included lectures, mock interviews, role play and field practice at the community level. A multilayer monitoring system was employed to maintain the quality of data and several tasks such as spot checking, thorough checking and back checking of the questionnaire were performed. Any discrepancies arising were resolved through re-interviewing the respective mothers.

\section{Variables assessed and measured}

Child characteristics considered for the present study were: age in months; gender (boy/girl); birth order (1-2, $3-4, \geq 5)$; birth weight in kilograms $(<2 \cdot 5, \geq 2 \cdot 5)$; immediate breast-feeding after birth (yes/no); prelacteal feeding (yes/no); exclusive breast-feeding up to 6 months (yes/no); and suffering from diarrhoea within the last 3 months (yes/no). Although the study did not collect data on exact birth weight, mothers were asked to report, according to their perception, whether their child was 'very small', 'smaller than average', 'average', 'larger than average' or 'very large' at birth. A birth size of 'very small' or 'smaller than average' was categorized as low birth weight $(<2.5 \mathrm{~kg})$, while a birth size of 'average' to 'very large' was categorized as normal birth weight $(\geq 2 \cdot 5 \mathrm{~kg})$. Maternal characteristics were: age in years; level of education (no education, primary incomplete: grade 1-4, primary complete/secondary incomplete: grade 5-9, 


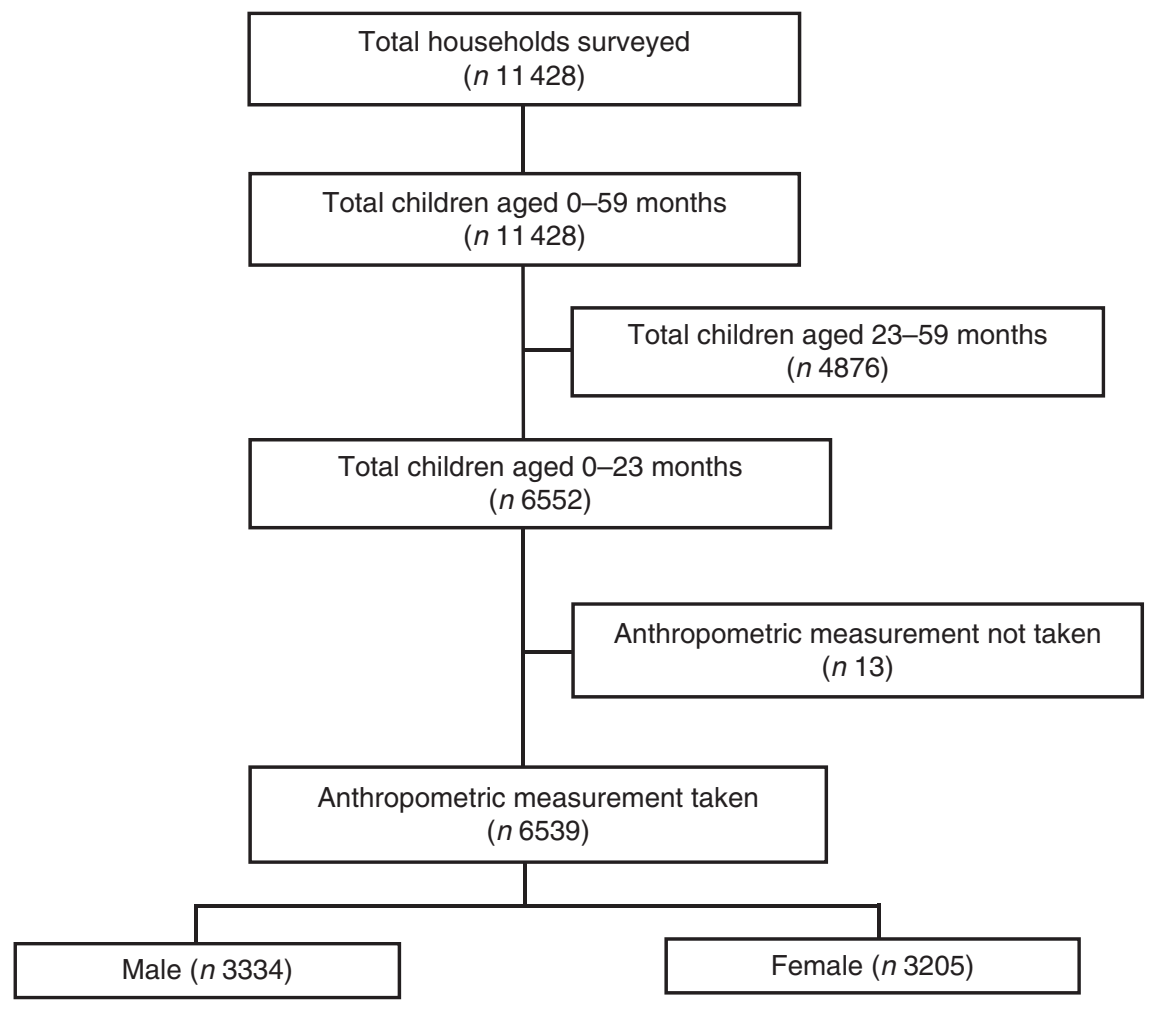

Fig. 1 Study profile and enrolment of participants

secondary or higher: grade 10 or higher); occupation (housewife/working outside), BMI in $\mathrm{kg} / \mathrm{m}^{2}$ (underweight: $<18 \cdot 5$, normal: $18 \cdot 5-24 \cdot 9$, overweight: $25 \cdot 0-29 \cdot 9$, obese: $\geq 30 \cdot 0)$, age at first pregnancy in years $(<20, \geq 20)$; vitamin A supplementation immediately after delivery (yes/no); Fe supplementation during pregnancy (yes/no); using soap before eating (yes/no); and using soap after defecation (yes/no). Household-level characteristics were: administrative division (Barisal, Chittagong, Dhaka, Khulna, Rajshahi, Rangpur, Sylhet); place of residence (rural areas/urban slums); household wealth quintile (lowest, low, middle, high, highest); size of household $(\leq 4,>4)$; household food security status (secure/insecure); household source of drinking-water (safe/unsafe); type of latrine (improved/unimproved); and toilet hygiene condition (hygienic/unhygienic).

Construction of the wealth index was based on factor analysis of principal component analysis of key socioeconomic variables ${ }^{(26)}$. The variables considered were: type of wall, floor and roof of the house; ownership of a radio, television, computer, bicycle, mobile/telephone, refrigerator, wardrobe, table, chair, watch, bed, sewing machine, bike, motor vehicle and livestock; and access to solar electricity.

Age of the child was recorded from the immunization card or birth certificate. A local event calendar was used when this information was unavailable. Height was measured to the nearest $0 \cdot 1 \mathrm{~cm}$ using a board with a wooden base and a movable headpiece, with the participant in supine position. Weight was measured with an electronic bathroom scale at $0 \cdot 1 \mathrm{~kg}$ precision. Height and weight of the children and mothers were taken separately. BMI of the mothers was calculated using the formula ${ }^{(27)}$ : [weight $(\mathrm{kg})] /[\text { height }(\mathrm{m})]^{2}$.

The UNICEF and WHO Joint Monitoring Programme's operational definition for improved water supply, treated water and improved sanitation facilities was used ${ }^{(28)}$. The households were considered food insecure if they faced any food insufficiencies in the $30 \mathrm{~d}$ prior to the survey.

\section{Outcome measurement}

The outcome variable was stunting of children aged 0-23 months. A child with height-for-age more than 2 sD below the median height-for-age of the WHO reference population (height-for-age $Z$-score $<-2$ ) was considered stunted $^{(29)}$.

\section{Statistical analysis}

Descriptive analysis was performed to assess the distribution of the variables. The $\chi^{2}$ test was used to compare the prevalence of stunting within different categories of a variable with 5\% level of significance. As the EA were scattered throughout the country with different demographic and social characteristics, there was a potential chance for certain variability in stunting prevalence among clusters. Moreover, mothers from the same EA could share certain types of unobserved cultural and environmental 
factors. This nested source of variability was addressed by incorporating random effects with the usual fixed effects in the model ${ }^{(30)}$. Therefore, considering EA as clusters, the modified Poisson regression was executed to explore the factors truly associated with early childhood stunting ${ }^{(31)}$. EA were considered as random effects with exchangeable correlation among clusters, while other variables were considered as fixed effects. The parameters of the model were estimated through the generalized estimating equations approach to estimate the true effect of associated factors and the unknown correlation of the outcome among clusters ${ }^{(32)}$. Due to the convergence problem with many covariates in the binomial model, the modified Poisson regression model was performed, which is equivalent to the binomial model while estimating the risk ratio ${ }^{(31)}$. The crude risk ratio (cRR), adjusted risk ratio (aRR) and 95\% CI were estimated at 5\% level of significance. The children's anthropometric index was constructed in WHO AnthroPlus software and all statistical analyses were performed using the statistical software package Stata version 13.0.

\section{Etbical approval}

The guidelines of the Declaration of Helsinki were followed while conducting the study and ethical clearance was sought from the Bangladesh Medical Research Council (reference number BMRC/NREC/2013-2016/802) before the study. Both written and verbal consent were taken from the mothers of the children prior to the interview.

\section{Results}

\section{Sample description}

Of the 6539 children less than 2 years of age sampled, $23.7 \%$ were $0-5$ months old, $31 \cdot 1 \%$ were 6-11 months and $45 \cdot 2 \%$ were $12-23$ months old (Table 1 ). The male: female ratio was close to unity $(49.0$ v. $51 \cdot 0 \%$, respectively). Meanwhile, $63.4 \%$ of mothers were $20-29$ years of age and $20.5 \%$ were aged 30 years or above. In addition, $13.6 \%$ of children's mothers had no education while $14.3 \%$ had completed grade 10 or higher. Most of the mothers $(94.4 \%)$ were housewives. Also, $20.6 \%$ of the mothers were underweight, $14.4 \%$ were overweight and only $2 \cdot 2 \%$ were obese. More than one-third of children were sampled from Dhaka division and $86.9 \%$ lived in rural areas. A total of $40.0 \%$ of children belonged to poor households (20.2\% lowest, $19 \cdot 8 \%$ low; Table 1$)$.

\section{Prevalence of childbood stunting}

Overall, 29.9\% of children were stunted (Table 2). The prevalence of stunting increased with age of the child $(P<0.001)$ and was higher among boys than girls $(32.6 v$. $27.1 \% ; P<0.001)$. The prevalence increased with higher
Table 1 Demographic and socio-economic characteristics of the sample of Bangladeshi children under 2 years old ( $n 6539)$, October 2015-January 2016

\begin{tabular}{|c|c|c|}
\hline Characteristic & $n$ & $\%$ \\
\hline \multicolumn{3}{|l|}{ Child's age (months) } \\
\hline $0-5$ & 1550 & $23 \cdot 70$ \\
\hline $6-11$ & 2032 & 31.08 \\
\hline $12-23$ & 2957 & $45 \cdot 22$ \\
\hline \multicolumn{3}{|l|}{ Child's gender } \\
\hline Female & 3205 & 49.01 \\
\hline Male & 3334 & 50.99 \\
\hline \multicolumn{3}{|l|}{ Maternal age (years) } \\
\hline$<20$ & 1055 & $16 \cdot 13$ \\
\hline $20-29$ & 4147 & 63.42 \\
\hline$\geq 30$ & 1337 & 20.45 \\
\hline \multicolumn{3}{|l|}{ Maternal education } \\
\hline No education & 888 & 13.58 \\
\hline Primary incomplete* & 1109 & $16 \cdot 96$ \\
\hline Primary or secondary incomplete† & 3605 & $55 \cdot 13$ \\
\hline Secondary or higher & 937 & 14.33 \\
\hline \multicolumn{3}{|l|}{ Maternal occupation } \\
\hline Housewife & 6175 & 94.43 \\
\hline Working outside & 364 & 5.57 \\
\hline \multicolumn{3}{|l|}{ Maternal BMI $\left(\mathrm{kg} / \mathrm{m}^{2}\right)$} \\
\hline$<18.5$ & 1348 & $20 \cdot 61$ \\
\hline $18 \cdot 5-24.9$ & 4102 & $62 \cdot 73$ \\
\hline $25 \cdot 0-29.9$ & 944 & 14.44 \\
\hline$\geq 30.0$ & 145 & $2 \cdot 22$ \\
\hline \multicolumn{3}{|l|}{ Division } \\
\hline Barisal & 453 & 6.93 \\
\hline Chittagong & 746 & 11.41 \\
\hline Dhaka & 2435 & $37 \cdot 24$ \\
\hline Khulna & 859 & $13 \cdot 14$ \\
\hline Rajshahi & 745 & 11.39 \\
\hline Rangpur & 801 & $12 \cdot 25$ \\
\hline Sylhet & 500 & 7.65 \\
\hline \multicolumn{3}{|l|}{ Place of residence } \\
\hline Rural & 5679 & $86 \cdot 85$ \\
\hline Urban slum & 860 & $13 \cdot 15$ \\
\hline \multicolumn{3}{|l|}{ Household wealth quintile } \\
\hline Lowest & 1323 & $20 \cdot 23$ \\
\hline Low & 1297 & 19.83 \\
\hline Middle & 1268 & $19 \cdot 39$ \\
\hline High & 1322 & $20 \cdot 22$ \\
\hline Highest & 1329 & $20 \cdot 32$ \\
\hline$n$ & 6539 & $100 \cdot 0$ \\
\hline
\end{tabular}

${ }^{*}$ Primary incomplete $=$ grade $1-4$.

†Primary or secondary incomplete $=$ grade $5-9$.

$\ddagger$ Secondary or higher $=$ grade 10 or higher.

birth order $(29 \cdot 2 \%$ for the first or second child, $30 \cdot 4 \%$ for the third or fourth child, and $37.2 \%$ for the fifth or later child; $P=0.006)$. Stunting prevalence was also significantly higher among low-birth-weight babies (weight $<2.5 \mathrm{~kg}$ ) than babies with normal birth weight (42.0 $v$. $29.0 \% ; P<0.001)$. The study further showed that the prevalence of stunting was considerably lower among children who were exclusively breast-fed up to 6 months compared with those who were not $(17 \cdot 1$ v. $32.9 \%$; $P<0 \cdot 001)$. Children suffering from diarrhoea episodes within the last 3 months were also more likely to be stunted (34.7 v. 29.4\%; $P=0.006)$.

Although not significant, the prevalence of childhood stunting was found to be increasing with mother's age. However, in contrast, the prevalence of stunting decreased significantly with increasing maternal education 
Table 2 Bivariate analysis of child, maternal and household characteristics with childhood stunting in Bangladeshi children under 2 years old ( $n$ 6539), October 2015-January 2016

\begin{tabular}{|c|c|c|c|}
\hline \multirow[b]{2}{*}{ Characteristic } & \multicolumn{2}{|c|}{ Stunting } & \multirow[b]{2}{*}{$P$ value } \\
\hline & $n$ & $\%$ & \\
\hline \multicolumn{4}{|l|}{ Child characteristics } \\
\hline \multicolumn{4}{|l|}{ Age (months) } \\
\hline $0-5$ & 1550 & $15 \cdot 42$ & \multirow[t]{3}{*}{$<0.001$} \\
\hline $6-11$ & 2032 & $21 \cdot 70$ & \\
\hline $12-23$ & 2957 & $43 \cdot 19$ & \\
\hline \multicolumn{4}{|l|}{ Gender } \\
\hline Female & 3205 & $27 \cdot 11$ & \multirow[t]{2}{*}{$<0.001$} \\
\hline Male & 3334 & 32.63 & \\
\hline \multicolumn{4}{|l|}{ Birth order } \\
\hline $1-2$ & 4438 & $29 \cdot 18$ & \multirow[t]{3}{*}{0.006} \\
\hline $3-4$ & 1746 & $30 \cdot 36$ & \\
\hline$\geq 5$ & 355 & $37 \cdot 18$ & \\
\hline \multicolumn{4}{|l|}{ Birth weight $(\mathrm{kg})$} \\
\hline$<2.5$ & 476 & $42 \cdot 02$ & \multirow[t]{2}{*}{$<0.001$} \\
\hline$\geq 2.5$ & 6063 & 28.98 & \\
\hline \multicolumn{4}{|c|}{ Breast-feeding immediately after birth } \\
\hline No & 938 & $30 \cdot 38$ & 0.742 \\
\hline Yes & 5601 & $29 \cdot 85$ & \\
\hline Prelacteal feeding & & & \\
\hline No & 3179 & $29 \cdot 10$ & 0.154 \\
\hline Yes & 3360 & $30 \cdot 71$ & \\
\hline Exclusive breast-feed & up to 6 & nths & \\
\hline No & 5319 & $32 \cdot 88$ & $<0.001$ \\
\hline Yes & 1220 & 17.05 & \\
\hline Diarrhoea within $3 \mathrm{~m}$ & & & \\
\hline No & 5923 & $29 \cdot 43$ & 0.006 \\
\hline Yes & 616 & 34.74 & \\
\hline Maternal characteristics & & & \\
\hline Age (years) & & & \\
\hline$<20$ & 1055 & 28.06 & 0.111 \\
\hline $20-29$ & 4147 & 29.76 & \\
\hline$\geq 30$ & 1337 & 31.94 & \\
\hline Education & & & \\
\hline No education & 888 & 38.74 & $<0.001$ \\
\hline Grade 1-4 & 1109 & 34.99 & \\
\hline Grade 5-9 & 3605 & $28 \cdot 27$ & \\
\hline Grade 10 or more & 937 & 21.99 & \\
\hline Occupation & & & \\
\hline Housewife & 6175 & 29.89 & 0.808 \\
\hline Working outside & 364 & 30.49 & \\
\hline BMl $\left(\mathrm{kg} / \mathrm{m}^{2}\right)$ & & & \\
\hline$<18.5$ & 1348 & 35.53 & $<0.001$ \\
\hline $18.5-24.9$ & 4102 & $29 \cdot 28$ & \\
\hline $25 \cdot 0-29.9$ & 944 & 25.42 & \\
\hline$\geq 30.0$ & 145 & $25 \cdot 52$ & \\
\hline Age at first pregnanc & ears) & & \\
\hline$<20$ & 4873 & $30 \cdot 70$ & 0.020 \\
\hline$\geq 20$ & 1666 & $27 \cdot 67$ & \\
\hline Vitamin A supplemen & & & \\
\hline No & 3208 & $31 \cdot 14$ & 0.036 \\
\hline Yes & 3331 & $28 \cdot 76$ & \\
\hline Fe supplementation & & & \\
\hline No & 1591 & 33.69 & $<0.001$ \\
\hline Yes & 4948 & $28 \cdot 72$ & \\
\hline Using soap before ea & & & \\
\hline No & 5223 & $30 \cdot 75$ & 0.003 \\
\hline Yes & 1316 & $26 \cdot 67$ & \\
\hline Using soap after defe & & & \\
\hline No & 1530 & $33 \cdot 73$ & $<0.001$ \\
\hline Yes & 5009 & $28 \cdot 77$ & \\
\hline Household characteristi & & & \\
\hline Division & & & \\
\hline Barisal & 453 & $26 \cdot 05$ & $<0.001$ \\
\hline Chittagong & 746 & 29.49 & \\
\hline Dhaka & 2435 & 31.62 & \\
\hline Khulna & 859 & 24.45 & \\
\hline
\end{tabular}

Table 2 Continued

\begin{tabular}{|c|c|c|c|}
\hline \multirow[b]{2}{*}{ Characteristic } & \multicolumn{2}{|c|}{ Stunting } & \multirow[b]{2}{*}{$P$ value } \\
\hline & $n$ & $\%$ & \\
\hline Rajshahi & 745 & $26 \cdot 85$ & \\
\hline Rangpur & 801 & 28.96 & \\
\hline Sylhet & 500 & 41.40 & \\
\hline \multicolumn{4}{|c|}{ Place of residence } \\
\hline Rural & 5679 & 29.57 & 0.099 \\
\hline Urban slum & 860 & $32 \cdot 33$ & \\
\hline \multicolumn{4}{|l|}{ Wealth quintile } \\
\hline Lowest & 1323 & $33 \cdot 18$ & $<0.001$ \\
\hline Low & 1297 & 34.77 & \\
\hline Middle & 1268 & $28 \cdot 79$ & \\
\hline High & 1322 & 28.06 & \\
\hline Highest & 1329 & 24.91 & \\
\hline \multicolumn{4}{|l|}{ Household size } \\
\hline$\leq 4$ & 2482 & $29 \cdot 65$ & 0.704 \\
\hline$>4$ & 4057 & $30 \cdot 10$ & \\
\hline \multicolumn{4}{|c|}{ Food security status } \\
\hline Insecure & 1673 & 34.91 & $<0.001$ \\
\hline Secure & 4866 & $28 \cdot 22$ & \\
\hline \multicolumn{4}{|c|}{ Drinking-water safe } \\
\hline No & 6049 & $30 \cdot 07$ & 0.375 \\
\hline Yes & 490 & $28 \cdot 16$ & \\
\hline \multicolumn{4}{|l|}{ Type of latrine } \\
\hline Unimproved & 5067 & 31.42 & $<0.001$ \\
\hline Improved & 1472 & $24 \cdot 80$ & \\
\hline \multicolumn{4}{|c|}{ Toilet hygiene condition } \\
\hline Unhygienic & 5155 & 31.79 & $<0.001$ \\
\hline Hygienic & 1384 & 22.98 & \\
\hline$n$ & 6539 & 29.93 & \\
\hline
\end{tabular}

$(P<0.001)$. A total of $38.7 \%$ of children whose mothers had no education were stunted, compared with 28.3 and $22.0 \%$, respectively, among children whose mothers had completed grade 5-9 and grade 10 or more. Maternal nutritional status, measured by BMI, also predicted decreased childhood stunting $(P<0 \cdot 001)$. Also, the prevalence of stunting was higher among children whose mothers became pregnant for the first time before the age of 20 years $(P=0.020)$. At the same time, the prevalence of stunting was also significantly lower among children whose mother received vitamin A supplements immediately after delivery $(P=0.036)$, received Fe supplements during pregnancy $(P<0 \cdot 001)$, used soap before eating $(P=0.003)$ and used soap after defecation $(P<0 \cdot 001)$.

Disparity in childhood stunting prevalence was observed among households from different administrative divisions $(P<0 \cdot 001)$. The prevalence was highest in Sylhet division (41.4\%) and lowest in Khulna (24.5\%). Also, the prevalence was higher among the children from urban slums than among those from rural areas (32.3 v. 29.6\%; $P=0.099$ ). Stunting prevalence was significantly higher among children from poor households (poorest: $33.2 \%$, richest: $24.9 \% ; P<0.001)$. However, the prevalence was considerably lower among children from food-secure households $(P<0.001)$, and among households with improved $(P<0.001)$ and hygienic $(P<0.001)$ latrines (Table 2). 
Risk factors for childbood stunting

Individual assessments of each of the covariates with childhood stunting are presented in Table 3. Significant associations were observed between stunting and different child characteristics such as age, gender, birth order, birth weight, exclusive breast-feeding up to 6 months and diarrhoea episodes. Maternal characteristics such as level of education, BMI, age at first pregnancy, Fe supplementation during pregnancy, use of soap before eating and after defecation were also significantly associated with childhood stunting. Moreover, household-level factors such as administrative division, wealth quintile, food security status, type of latrine and toilet hygiene condition were significantly associated with childhood stunting.

The final adjusted modified Poisson regression model captured $3 \%$ of variation $(P<0 \cdot 001)$ in outcome among clusters. The adjusted estimated effects for the factors associated with childhood stunting are also presented in
Table 3. We found that the children aged 6-11 months had $34 \%$ higher risk of stunting than those aged 0-5 months (aRR $=1.34 ; 95 \%$ CI 1.11, 1.62), while children aged 1223 months had 2.65 times higher risk $(\mathrm{aRR}=2 \cdot 65 ; 95 \% \mathrm{CI}$ $2 \cdot 20,3 \cdot 20$ ). Meanwhile, boys had $21 \%$ higher risk than girls $(\mathrm{aRR}=1 \cdot 21 ; 95 \% \mathrm{CI} 1 \cdot 12,1 \cdot 30)$. It was also notable that low-birth-weight babies had more than $50 \%$ higher risk of stunting than babies with normal birth weight (aRR $=1.51 ; 95 \%$ CI $1.35,1.68)$.

The risk of stunting was $12 \%$ lower among children whose mothers had completed grade 5-9 $(\mathrm{aRR}=0 \cdot 88$; $95 \%$ CI $0 \cdot 80,0 \cdot 97$ ) and was $22 \%$ lower among children of mothers who had completed grade 10 or more $(\mathrm{aRR}=$ 0.78 ; $95 \%$ CI $0.67,0.92$ ) compared with the children of uneducated mothers. The children of underweight mothers had $11 \%$ higher risk of stunting than those of normal-weight mothers (aRR $=1.11 ; 95 \%$ CI $1.02,1.20$ ). Meanwhile, there was 9\% higher risk of stunting among

Table 3 Association of child, maternal and household characteristics with childhood stunting in Bangladeshi children under 2 years old (n 6539), October 2015-January 2016

\begin{tabular}{|c|c|c|c|c|c|c|}
\hline \multirow[b]{2}{*}{ Characteristic } & \multicolumn{3}{|c|}{ Crude } & \multicolumn{3}{|c|}{ Adjusted } \\
\hline & RR & $95 \% \mathrm{Cl}$ & $P$ value & RR & $95 \% \mathrm{Cl}$ & $P$ value \\
\hline \multicolumn{7}{|l|}{ Child characteristics } \\
\hline \multicolumn{7}{|l|}{ Age (months) } \\
\hline $0-5$ & 1.00 & Ref. & - & 1.00 & Ref. & - \\
\hline $6-11$ & 1.40 & $1.18,1.64$ & $<0.001$ & 1.34 & $1.11,1.62$ & 0.003 \\
\hline $12-23$ & 2.77 & $2.37,3.23$ & $<0.001$ & 2.65 & $2 \cdot 20,3 \cdot 20$ & $<0.001$ \\
\hline \multicolumn{7}{|l|}{ Gender } \\
\hline Female & 1.00 & Ref. & - & 1.00 & Ref. & - \\
\hline Male & 1.20 & $1.11,1.30$ & $<0.001$ & 1.21 & $1.12,1.30$ & $<0.001$ \\
\hline \multicolumn{7}{|l|}{ Birth order } \\
\hline $1-2$ & 1.00 & Ref. & - & 1.00 & Ref. & - \\
\hline $3-4$ & 1.02 & $0.94,1.12$ & 0.593 & 0.94 & $0.85,1.03$ & 0.195 \\
\hline$\geq 5$ & 1.19 & $1.02,1.39$ & 0.029 & 0.96 & $0.81,1.14$ & 0.624 \\
\hline \multicolumn{7}{|l|}{ Birth weight $(\mathrm{kg})$} \\
\hline$\geq 2.5$ & 1.00 & Ref. & - & 1.00 & Ref. & - \\
\hline$<2.5$ & 1.51 & $1.35,1.69$ & $<0.001$ & 1.51 & $1.35,1.68$ & $<0.001$ \\
\hline \multicolumn{7}{|c|}{ Breast-feeding immediately after birth } \\
\hline No & 1.00 & Ref. & - & 1.00 & Ref. & - \\
\hline Yes & 0.96 & $0.87,1.07$ & 0.454 & 1.05 & $0.95,1.16$ & 0.337 \\
\hline \multicolumn{7}{|l|}{ Prelacteal feeding } \\
\hline No & 1.00 & Ref. & - & 1.00 & Ref. & _- \\
\hline Yes & 1.02 & $0.95,1.10$ & 0.524 & 1.03 & $0.96,1.11$ & 0.458 \\
\hline \multicolumn{7}{|c|}{ Exclusive breast-feeding up to 6 months } \\
\hline No & 1.00 & Ref. & - & 1.00 & Ref. & - \\
\hline Yes & 0.51 & $0.44,0.59$ & $<0.001$ & 0.96 & $0.81,1.15$ & 0.664 \\
\hline \multicolumn{7}{|c|}{ Diarrhoea within 3 months } \\
\hline No & 1.00 & Ref. & - & 1.00 & Ref. & - \\
\hline Yes & 1.15 & $1.03,1.28$ & 0.011 & 1.02 & $0.92,1.14$ & 0.662 \\
\hline \multicolumn{7}{|l|}{ Maternal characteristics } \\
\hline \multicolumn{7}{|l|}{ Age (years) } \\
\hline$<20$ & 1.00 & Ref. & - & 1.00 & Ref. & - \\
\hline $20-29$ & 0.94 & $0.84,1.06$ & 0.338 & 0.97 & $0.86,1 \cdot 10$ & 0.622 \\
\hline$\geq 30$ & 1.05 & $0.96,1.15$ & 0.279 & 1.05 & $0.94,1.17$ & 0.413 \\
\hline \multicolumn{7}{|l|}{ Edūcation } \\
\hline No education & 1.00 & Ref. & _- & 1.00 & Ref. & _- \\
\hline Grade $1-4$ & 0.93 & $0.83,1.04$ & 0.221 & 0.99 & $0 \cdot 88,1 \cdot 10$ & 0.798 \\
\hline Grade 5-9 & 0.76 & $0.69,0.84$ & $<0.001$ & 0.88 & $0.80,0.97$ & 0.009 \\
\hline Grade 10 or more & 0.61 & $0.52,0.70$ & $<0.001$ & 0.78 & $0.67,0.92$ & 0.003 \\
\hline \multicolumn{7}{|l|}{ Occupation } \\
\hline Housewife & 1.00 & Ref. & - & 1.00 & Ref. & _- \\
\hline Working outside & 1.02 & $0.89,1.18$ & 0.755 & 1.00 & $0.87,1.16$ & 0.966 \\
\hline
\end{tabular}




\begin{tabular}{|c|c|c|c|c|c|c|}
\hline \multirow[b]{2}{*}{ Characteristic } & \multicolumn{3}{|c|}{ Crude } & \multicolumn{3}{|c|}{ Adjusted } \\
\hline & RR & $95 \% \mathrm{Cl}$ & $P$ value & $\mathrm{RR}$ & $95 \% \mathrm{Cl}$ & $P$ value \\
\hline \multicolumn{7}{|l|}{ BMI $\left(\mathrm{kg} / \mathrm{m}^{2}\right)$} \\
\hline$<18.5$ & 1.19 & $1.10,1.30$ & $<0.001$ & 1.11 & $1.02,1.20$ & 0.011 \\
\hline $18.5-24.9$ & 1.00 & Ref. & - & 1.00 & Ref. & - \\
\hline $25 \cdot 0-29 \cdot 9$ & 0.89 & $0.79,1.02$ & 0.090 & 0.96 & $0.85,1.09$ & 0.541 \\
\hline$\geq 30.0$ & 0.91 & $0.69,1.18$ & 0.472 & 1.02 & $0.78,1.32$ & 0.909 \\
\hline \multicolumn{7}{|c|}{ Age at first pregnancy (years) } \\
\hline$\geq 20$ & 1.00 & Ref. & - & 1.00 & Ref. & - \\
\hline$<20$ & 1.13 & $1.03,1.24$ & 0.012 & 1.09 & $0.99,1.19$ & 0.097 \\
\hline \multicolumn{7}{|c|}{ Vitamin A supplementation } \\
\hline No & 1.00 & Ref. & - & 1.00 & Ref. & - \\
\hline Yes & 0.94 & $0.87,1.02$ & 0.165 & 1.01 & $0.93,1.09$ & 0.857 \\
\hline \multicolumn{7}{|c|}{ Fe supplementation } \\
\hline No & 1.00 & Ref. & - & 1.00 & Ref. & - \\
\hline Yes & 0.86 & $0.80,0.94$ & $<0.001$ & 0.96 & $0.89,1.04$ & 0.343 \\
\hline \multicolumn{7}{|c|}{ Using soap before eating } \\
\hline No & 1.00 & Ref. & - & 1.00 & Ref. & - \\
\hline Yes & 0.86 & $0.78,0.96$ & 0.005 & 0.95 & $0.86,1.06$ & 0.366 \\
\hline \multicolumn{7}{|c|}{ Using soap after defecation } \\
\hline No & 1.00 & Ref. & - & 1.00 & Ref. & - \\
\hline Yes & 0.87 & $0.81,0.94$ & 0.001 & 1.01 & $0.93,1.09$ & 0.883 \\
\hline \multicolumn{7}{|c|}{ Household characteristics } \\
\hline \multicolumn{7}{|c|}{ Division } \\
\hline Barisal & 0.84 & $0.69,1.04$ & 0.108 & 0.89 & $0.74,1.07$ & 0.217 \\
\hline Chittagong & 0.94 & $0.81,1.08$ & 0.384 & 0.98 & $0 \cdot 84,1 \cdot 14$ & 0.779 \\
\hline Dhaka & 1.00 & Ref. & - & 1.00 & Ref. & - \\
\hline Khulna & 0.78 & $0.65,0.93$ & 0.007 & 0.84 & $0.72,0.99$ & 0.038 \\
\hline Rajshahi & 0.87 & $0.73,1.03$ & 0.116 & 0.93 & $0.79,1 \cdot 10$ & 0.404 \\
\hline Rangpur & 0.93 & $0 \cdot 78,1 \cdot 11$ & 0.411 & 0.97 & $0.83,1.13$ & 0.708 \\
\hline Sylhet & 1.34 & $1.07,1.68$ & 0.011 & 1.35 & $1.12,1.64$ & 0.002 \\
\hline \multicolumn{7}{|c|}{ Place of residence } \\
\hline Rural & 1.00 & Ref. & - & 1.00 & Ref. & - \\
\hline \multirow{2}{*}{\multicolumn{7}{|c|}{ Wealth quintile }} \\
\hline & & & & & & \\
\hline Lowest & 1.00 & Ref. & - & 1.00 & Ref. & - \\
\hline Low & 1.02 & $0.91,1 \cdot 13$ & 0.748 & 1.03 & $0.93,1.15$ & 0.546 \\
\hline Middle & 0.85 & $0.76,0.94$ & 0.002 & 0.90 & $0.81,1.01$ & 0.063 \\
\hline High & 0.83 & $0.73,0.94$ & 0.003 & 0.93 & $0.82,1.05$ & 0.231 \\
\hline Highest & 0.68 & $0.59,0.79$ & $<0.001$ & 0.84 & $0.72,0.98$ & 0.030 \\
\hline \multicolumn{7}{|l|}{ Household size } \\
\hline$\leq 4$ & 1.00 & Ref. & - & 1.00 & Ref. & - \\
\hline$>4$ & 0.98 & $0.90,1.06$ & 0.593 & 1.03 & $0.95,1.13$ & 0.436 \\
\hline \multicolumn{7}{|c|}{ Food security status } \\
\hline Insecure & 1.00 & Ref. & - & 1.00 & Ref. & - \\
\hline Secure & 0.81 & $0.74,0.88$ & $<0.001$ & 0.93 & $0.85,1.00$ & 0.064 \\
\hline \multicolumn{7}{|c|}{ Drinking-water safe } \\
\hline No & 1.00 & Ref. & - & 1.00 & Ref. & - \\
\hline \multirow{2}{*}{\multicolumn{7}{|c|}{ Type of latrine }} \\
\hline & & & & & & \\
\hline Unimproved & 1.00 & Ref. & - & 1.00 & Ref. & - \\
\hline Improved & 0.76 & $0.69,0.84$ & $<0.001$ & 0.88 & $0.79,0.98$ & 0.025 \\
\hline \multicolumn{7}{|c|}{ Toilet hygiene condition } \\
\hline Unhygienic & 1.00 & Ref. & - & 1.00 & Ref. & - \\
\hline Hygienic & 0.74 & $0.66,0.82$ & $<0.001$ & 0.90 & $0.79,1.02$ & 0.086 \\
\hline
\end{tabular}

$R R$, risk ratio; Ref., reference category.

children whose mothers became pregnant for the first time before the age of 20 years ( $\mathrm{aRR}=1.09$; $95 \%$ CI 0.99, 1.19).

It was found that children from Khulna division had $16 \%$ lower risk of stunting compared with those of Dhaka (aRR $=0.84 ; 95 \%$ CI 0.72, 0.99), while the risk was 35\% higher among children from Sylhet division $(\mathrm{aRR}=1.35$; $95 \%$ CI $1 \cdot 12,1.64)$. It was also evident that children from urban slums had $19 \%$ higher risk of stunting than those from rural areas $(\mathrm{aRR}=1 \cdot 19 ; 95 \%$ CI 1.00, 1.41). Also, children from the richest households had 16\% lower risk of stunting than those from the poorest households $(\mathrm{aRR}=0.84 ; 95 \%$ CI $0.72,0.98)$. Moreover, children from food-secure households had $7 \%$ lower risk $(\mathrm{aRR}=0.93$; $95 \%$ CI $0 \cdot 85,1 \cdot 00)$, households having improved toilet had $12 \%$ lower risk (aRR $=0.88 ; 95 \%$ CI $0.79,0.98)$ and households having hygienic toilet had $10 \%$ lower risk (aRR $=0.90 ; 95 \%$ CI $0.79,1.02$ ) of stunting compared with their corresponding reference (Table 3 ). 


\section{Discussion}

The present study recognized several modifiable risk factors of stunting at individual, maternal and household levels among children aged 0-23 months in Bangladesh. Although the overall stunting prevalence among children has been declining in Bangladesh over the last decade, the reduction rate is not satisfactory ${ }^{(12)}$. The findings of the present study suggest that an intervention considering multilevel factors might increase the reduction rate, which in turn would help to achieve the World Health Assembly targets on reducing childhood stunting.

Overall, about one-third of children were stunted and there was marked variation in stunting prevalence among children based on place of residence, region and economic status. This agrees with the findings of the most recent national Demographic and Health Survey ${ }^{(12)}$. The present study also found that the prevalence of stunting was comparatively higher among the children from urban slums, which may be due to lack of adequate public services as well as health and sanitation facilities in slums. This is in line with the findings of some other studies ${ }^{(33-35)}$. Furthermore, as reported elsewhere ${ }^{(22)}$, stunting prevalence was considerably higher in Sylhet division, which is characterized by high rate of poverty, low literacy and lack of access to safe water and sanitation ${ }^{(36)}$.

High prevalence of stunting among young children can be attributed to chronic nutrition deprivation and poor linear growth in infancy and early childhood ${ }^{(37,38)}$. The demand for nutrients increases with age and inappropriate complementary feeding may result in inadequate intakes of vital nutrients, which is one of the major causes of stunting in early childhood ${ }^{(39,40)}$. However, evidence of the positive role of exclusive breast-feeding on linear growth is surprisingly weak ${ }^{(41)}$. The current study also found no significant association between exclusive breastfeeding and risk of early childhood stunting.

It is well documented in several studies ${ }^{(37,42)}$ that children born with a birth weight of less than $2.5 \mathrm{~kg}$ possess a higher risk of being stunted in early childhood. The present study also found that children born with low birth weight had $50 \%$ higher risk of being stunted. The association between low birth weight and stunting in childhood can be explained by the increased vulnerability of children to infections and increased risk of complications including sleep apnoea, jaundice, anaemia, chronic lung disorders, fatigue and loss of appetite ${ }^{(43,44)}$. As reported in an earlier study carried out in Bangladesh ${ }^{(37)}$, the present study also found that stunting prevalence was higher among boys than girls. The explanation for this gender variation in stunting is still unclear, although some researchers have argued that this disparity is more prevalent among poor households compared with those better-off socio-economically ${ }^{(45)}$.

Several maternal factors were also identified to be significantly associated with stunting among children under 2 years of age. It was evident that children of undernourished mothers were more likely to be stunted than those of well-nourished mothers. Maternal undernutrition is one of the major causes of intra-uterine growth retardation which contributes about $20 \%$ of the global burden of childhood stunting ${ }^{(3)}$. Moreover, stunted girls are likely to grow to be stunted women in their later life, which adversely affects growth of the fetus during their pregnancy. Suboptimal growth of the fetus often results in low birth weight at delivery ${ }^{(46)}$, which contributes to stunting in early childhood. However, the present study showed that maternal age at first pregnancy played a pivotal role in stunting among the children, which is also supported by other studies ${ }^{(37,47)}$. Furthermore, mother's education was identified as an important predictor of stunting. Studies conducted in Bangladesh ${ }^{(22,48)}$ and India ${ }^{(37)}$ also confirmed that higher maternal education significantly reduces stunting among children. Another multi-country study ${ }^{(49)}$ showed that $43 \%$ of the total reduction in stunting can be attributed to improvement in maternal education. Therefore, certain nutrition-sensitive and nutrition-specific interventions targeting adolescent girls and mothers may also be central to the national efforts in reducing childhood stunting and achieving the World Health Assembly target ${ }^{(6,19,50)}$. Since mothers are the main caregivers in households for young children, women's empowerment in terms of education and decision making should also be given attention when designing nutrition intervention programmes.

At household level, children from poor and foodinsecure households were more likely to be stunted ${ }^{(33)}$. This is similar to the findings of the most recent national Demographic and Health Survey ${ }^{(12)}$. Besides, lack of access to a sanitary latrine and unhygienic toilet condition were significantly associated with higher risk of stunting among children. This association is in line with the findings of some other previously conducted studies ${ }^{(51,52)}$. Therefore, to reduce stunting prevalence, policy makers should focus on reducing poverty and household food insecurity through ensuring easy access to credit, promoting small investments and social safety net transfers, subsidizing raw materials of production such as seeds and fertilizer, subsidizing the cost of medicines, and encouraging homestead gardening, among others.

However, merely implementing nutrition interventions may not necessarily ensure the desired level of reduction in childhood stunting prevalence in Bangladesh. It is also important that a suitable environment is being ensured where successful programme implementation can be achieved. A recent systematic review also highlighted that certain factors such as political stability, community mobilization, a multisectoral approach and programme delivery using community-based platforms can contribute to achieve higher rate of reduction ${ }^{(53)}$. It is also very crucial that the poorest of the poor ${ }^{(2)}$ and geographically vulnerable people ${ }^{(22)}$ are given the highest priority. 
We used the modified Poisson regression model with the generalized estimating equations approach in the present study while estimating the unknown correlation of outcome among clusters. Future research may also employ mixed-effect models as an alternative to using generalized estimating equations as mixed-effect models are capable of estimating the variance of multiple random or nested random effects ${ }^{(54)}$.

The present study has several strengths over other similar studies. First, it followed a rigorous sampling method covering a large sample size of children aged less than 2 years. Second, along with the rural areas, it also covered urban slums of the country where living standards as well as health and sanitation parameters are worse than in rural areas. Thus, the inclusion of children from urban slums alongside those from rural areas greatly increases the comprehensiveness of the study. However, the study was subjected to certain limitations too. Being crosssectional in nature, it was unable to draw out the temporal relationship between stunting and its determinants. Third, since the base study was carried out in rural areas and urban slums where BRAC operates its health and nutrition interventions, we were unable to include the children of urban non-slum areas of the country. Lastly, we had to rely on mother's perception of the size of her child at birth instead of the exact birth weight. This is due to the fact that birth weight is not being taken for many newborn babies, particularly in cases of home deliveries in Bangladesh.

\section{Conclusions}

The present study findings urge the importance of administering a multicomponent, multidisciplinary intervention to address the issue of stunting among children aged 0-23 months in Bangladesh. Policy makers and public health practitioners should focus on specific initiatives such as quality health-care services, promotion of breast-feeding, sanitation facilities, household food security and especially on maternal education and nutrition.

\section{Acknowledgements}

Acknowledgements: The authors acknowledge the role of the relevant staff of the Health Nutrition and Population Programme, BRAC, for initial review of the tools and smooth arrangement of the data collection initiatives. Financial support: This research was funded by the Strategic Partnership Arrangement (SPA) between BRAC, the UK Department for International Development (DFID) and the Australian Department of Foreign Affairs and Trade (DFAT). The funders had no role in the design, analysis or writing of this manuscript. Conflict of interest: The authors have no conflict of interest to disclose. Authorship: S.K.M., M.R. and K.A. contributed to the design of the study. M.B.H. conducted the data analysis and participated in interpretation of the results. S.K.M., M.B.H., M.P., F.K. and F.A. contributed to write the first draft of the manuscript. F.M.Y. commented extensively on the manuscript draft. All authors read and approved the final manuscript. Ethics of human subject participation: All procedures performed in studies involving human participants were in accordance with the ethical standards of the institutional and/or national research committee and with the 1964 Helsinki Declaration and its later amendments or comparable ethical standards. Ethical approval was obtained from the Bangladesh Medical Research Council (reference number BMRC/NREC/2013-2016/802). The purpose of the study was described and written informed consent was obtained from the child's parents prior to the interview. The respondents were ensured of the confidentiality of information provided.

\section{References}

1. Humphrey JH \& Prendergast AJ (2017) Population-level linear growth faltering in low-income and middle-income countries. Lancet Glob Health 5, e1168-e1169.

2. de Onis M \& Branca F (2016) Childhood stunting: a global perspective. Matern Child Nutr 12, 12-26.

3. Black RE, Victora CG, Walker SP et al. (2013) Maternal and child undernutrition and overweight in low-income and middle-income countries. Lancet 382, 427-451.

4. Stewart CP, Iannotti L, Dewey KG et al. (2013) Contextualising complementary feeding in a broader framework for stunting prevention. Matern Child Nutr 9, 27-45.

5. Martorell R \& Zongrone A (2012) Intergenerational influences on child growth and undernutrition. Paediatr Perinat Epidemiol 26, 302-314.

6. World Health Organization (2014) Global Nutrition Targets 2025: Policy Brief Series. Geneva: WHO.

7. The World Bank (2006) Repositioning Nutrition as Central to Development - A Strategy for Large-Scale Action. Washington, DC: World Bank.

8. Grantham-McGregor S, Cheung YB, Cueto S et al. (2007) Developmental potential in the first 5 years for children in developing countries. Lancet 369, 60-70.

9. Fenske N, Burns J, Hothorn T et al. (2013) Understanding child stunting in India: a comprehensive analysis of socioeconomic, nutritional and environmental determinants using additive quantile regression. PLoS One 8, e78692.

10. Goudet SM, Griffiths PL, Bogin BA et al. (2015) Nutritional interventions for preventing stunting in children (0 to 5 years) living in urban slums. Cochrane Database Syst Rev issue 5, CD011695; available at https://www.cochrane library.com/cdsr/doi/10.1002/14651858.CD011695/epdf/full

11. UNICEF, WHO \& World Bank Group (2017) Joint Child Malnutrition Estimates - Levels and Trends (2017 edition). http://www.who.int/nutgrowthdb/estimates2016/en/ (accessed October 2018).

12. National Institute of Population Research and Training, Mitra and Associates \& ICF International (2014) Bangladesh Demographic and Health Survey 2014. Dhaka and Rockville, MD: NIPORT, Mitra and Associates, and ICF International.

13. United Nations (2014) World Urbanization Prospects: The 2014 Revision. Washington, DC: UN, Department of Economic and Social Affairs, Population Division.

14. Bangladesh Bureau of Statistics (2015) Census of Slum Areas and Floating Population 2014. Dhaka: BBS. 
15. National Institute of Population Research and Training, International Centre for Diarrhoeal Disease Research Bangladesh \& MEASURE Evaluation (2015) Bangladesh Urban Health Survey 2013. Dhaka and Chapel Hill, NC: NIPORT, icddr,b and MEASURE Evaluation.

16. National Institute of Population Research and Training, International Centre for Diarrhoeal Disease Research Bangladesh, MEASURE Evaluation et al. (2008) Bangladesh Urban Health Survey 2006. Dhaka and Chapel Hill, NC: NIPORT, icddr,b and MEASURE Evaluation.

17. Ahsan KZ, El Arifeen S, Al-Mamun MA et al. (2017) Effects of individual, household and community characteristics on child nutritional status in the slums of urban Bangladesh. Arch Public Health 75, 9.

18. Bangladesh Bureau of Statistics \& UNICEF (2016) Child Well-Being Survey in Urban Areas of Bangladesh - Key Results. Dhaka: BBS \& UNICEF.

19. Ahmed T, Hossain M, Mahfuz M et al. (2016) Imperatives for reducing child stunting in Bangladesh. Matern Child Nutr 12, 242-245.

20. Fuchs C, Sultana T, Ahmed T et al. (2014) Factors associated with acute malnutrition among children admitted to a diarrhoea treatment facility in Bangladesh. Int J Pediatr 2014, 267806.

21. Fakir AMS \& Khan MWR (2015) Determinants of malnutrition among urban slum children in Bangladesh. Health Econ Rev 5, 1-11.

22. Hossain MB \& Khan MHR (2018) Role of parental education in reduction of prevalence of childhood undernutrition in Bangladesh. Public Health Nutr 21, 1845-1854.

23. Leroy JL, Ruel M, Habicht J-P et al. (2014) Linear growth deficit continues to accumulate beyond the first 1000 days in low- and middle-income countries: global evidence from 51 national surveys. J Nutr 144, 1460-1466.

24. DeRenzi B, Borriello G, Jackson J et al. (2011) Mobile phone tools for field-based health care workers in low-income countries. Mt Sinai J Med 78, 406-418.

25. Davis RE, Couper MP, Janz NK et al. (2010) Interviewer effects in public health surveys. Health Educ Res 25, 14-26.

26. Rutstein SO (2015) Steps to Constructing the New DHS Wealth Index. Rockville, MD: ICF International.

27. Gibson RS (2005) Principles of Nutritional Assessment. New York: Oxford University Press.

28. UNICEF \& World Health Organization (2015) Progress on Sanitation and Drinking Water: 2015 Update and MDG Assessment. https://www.unicef.org/publications/index_ 82419.html (accessed October 2018).

29. World Health Organization (2006) WHO Child Growth Standards: Length/Height for Age, Weight-for-Age, Weightfor-Length, Weight-for-Height and Body Mass Index-forAge, Methods and Development. Geneva: WHO.

30. Agresti A (2003) Categorical Data Analysis, vol. 482. New York: John Wiley \& Sons.

31. Zou GY \& Donner A (2013) Extension of the modified Poisson regression model to prospective studies with correlated binary data. Stat Methods Med Res 22, 661-670.

32. Zeger SL, Liang K-Y \& Albert PS (1988) Models for longitudinal data: a generalized estimating equation approach. Biometrics 44, 1049-1060.

33. Awasthi S \& Agarwal S (2003) Determinants of childhood mortality and morbidity in urban slums in India. Indian Pediatr 40, 1145-1161.

34. Ghosh S \& Shah D (2004) Nutritional problems in urban slum children. Indian Pediatr 41, 682-696.

35. Islam MM, Sanin KI, Mahfuz M et al. (2018) Risk factors of stunting among children living in an urban slum of Bangladesh: findings of a prospective cohort study. BMC Public Health 18, 197.

36. Center for Environmental and Geographic Information Services (2012) Master Plan of Haor Area, vol. I. Dhaka: Center for Environmental and Geographic Information Services.
37. Aguayo VM, Nair R, Badgaiyan N et al. (2016) Determinants of stunting and poor linear growth in children under 2 years of age in India: an in-depth analysis of Maharashtra's comprehensive nutrition survey. Matern Child Nutr 12, 121-140.

38. Jones AD, Ickes SB, Smith LE et al. (2014) World Health Organization infant and young child feeding indicators and their associations with child anthropometry: a synthesis of recent findings. Matern Child Nutr 10, 1-17.

39. Shrimpton R, Victora CG, de Onis M et al. (2001) Worldwide timing of growth faltering: implications for nutritional interventions. Pediatrics 107, e75-e75.

40. Dewey KG \& Adu-Afarwuah S (2008) Systematic review of the efficacy and effectiveness of complementary feeding interventions in developing countries. Matern Child Nutr 4, 24-85.

41. Bhutta ZA, Das JK, Rizvi A et al. (2013) Evidence-based interventions for improvement of maternal and child nutrition: what can be done and at what cost? Lancet 382, 452-477.

42. Mishra K, Kumar P, Basu S et al. (2014) Risk factors for severe acute malnutrition in children below 5 y of age in India: a case-control study. Indian J Pediatr 81, 762-765.

43. Haque SMR, Tisha S \& Huq N (2015) Poor birth size a badge of low birth weight accompanying less antenatal care in Bangladesh with substantial divisional variation: evidence from BDHS-2011. Public Health Res 5, 184-191.

44. Khanal V, Sauer K, Karkee R et al. (2014) Factors associated with small size at birth in Nepal: further analysis of Nepal Demographic and Health Survey 2011. BMC Pregnancy Childbirth 14, 32.

45. Wamani H, Astrøm AN, Peterson S et al. (2007) Boys are more stunted than girls in sub-Saharan Africa: a metaanalysis of 16 demographic and health surveys. BMC Pediatr 7, 17.

46. Young MF, Nguyen PH, Addo OY et al. (2015) The relative influence of maternal nutritional status before and during pregnancy on birth outcomes in Vietnam. Eur J Obstet Gynecol Reprod Biol 194, 223-227.

47. Varela-Silva MI, Azcorra H, Dickinson F et al. (2009) Influence of maternal stature, pregnancy age, and infant birth weight on growth during childhood in Yucatan, Mexico: a test of the intergenerational effects hypothesis. Am J Hum Biol 21, 657-663.

48. Headey D, Hoddinott J, Ali D et al. (2015) The other Asian enigma: explaining the rapid reduction of undernutrition in Bangladesh. World Dev 66, 749-761.

49. van den Bold M, Quisumbing AR \& Gillespie S (2013) Women's Empowerment and Nutrition: An Evidence Review (vol. 01294). Washington, DC: International Food Policy Research Institute; available at https://pdfs.semantic scholar.org/e43b/1432ddc7f87327fc0d086893da802df7a61a. pdf

50. Haddad L, Achadi E, Bendech MA et al. (2015) The Global Nutrition Report 2014: actions and accountability to accelerate the world's progress on nutrition. J Nutr 145, 663-671.

51. Fink G, Gunther I \& Hill K (2011) The effect of water and sanitation on child health: evidence from the demographic and health surveys 1986-2007. Int J Epidemiol 40, 1196-1204.

52. Rah JH, Cronin AA, Badgaiyan B et al. (2015) Household sanitation and personal hygiene practices are associated with child stunting in rural India: a cross-sectional analysis of surveys. BMJ Open 5, e005180.

53. Hossain M, Choudhury N, Abdullah KAB et al. (2017) Evidence-based approaches to childhood stunting in low and middle income countries: a systematic review. Arch Dis Child 102, 903-909.

54. Hubbard AE, Ahern J, Fleischer NL et al. (2010) To GEE or not to GEE: comparing population average and mixed models for estimating the associations between neighborhood risk factors and health. Epidemiology 21, 467-474. 